\title{
HIV Prevention Services and Testing Utilization Behaviors among Men Who Have Sex with Men at Elevated Risk for HIV in Chongqing, China
}

\author{
Dayong Huang, ${ }^{1}$ Yifei Hu, ${ }^{2}$ Guohui Wu, ${ }^{3}$ Yujiang Jia, ${ }^{4}$ Rongrong Lu, ${ }^{3}$ Yan Xiao, ${ }^{2}$ \\ H. F. Raymond, ${ }^{5}$ Willi McFarland, ${ }^{5,6}$ Yuhua Ruan, ${ }^{7}$ Wei Ma, ${ }^{8}$ and Jiangping Sun ${ }^{2}$ \\ ${ }^{1}$ Beijing Friendship Hospital, Capital Medical University, Beijing 100050, China \\ ${ }^{2}$ National Center for AIDS/STD Control and Prevention, Chinese Center for Disease Control and Prevention, \\ 155 Changbai Road, Changping District, Beijing 102206, China \\ ${ }^{3}$ Chongqing Center for Disease Control and Prevention, Chongqing 400042, China \\ ${ }^{4}$ Department of Preventive Medicine, Vanderbilt University School of Medicine, Nashville, TN 37232, USA \\ ${ }^{5}$ San Francisco Department of Public Health, San Francisco, CA 94102, USA \\ ${ }^{6}$ Department of Epidemiology and Biostatistics, University of California, San Francisco, CA 94105, USA \\ ${ }^{7}$ State Key Laboratory for Infectious Disease Prevention and Control, National Center for AIDS/STD Control and Prevention, \\ Chinese Center for Disease Control and Prevention, Collaborative Innovation Center for Diagnosis and Treatment of \\ Infectious Diseases, Beijing 102206, China \\ ${ }^{8}$ Shandong University School of Public Health, Jinan 250012, China \\ Correspondence should be addressed to Jiangping Sun; jpsun@chinaaids.cn
}

Received 13 October 2013; Revised 5 December 2013; Accepted 21 December 2013; Published 26 March 2014

Academic Editor: Muktar Aliyu

Copyright (c) 2014 Dayong Huang et al. This is an open access article distributed under the Creative Commons Attribution License, which permits unrestricted use, distribution, and reproduction in any medium, provided the original work is properly cited.

Objective. To investigate barriers and correlates of the use of HIV prevention services and HIV testing behaviors among men who have sex with men in Chongqing. Methods. Three consecutive cross-sectional surveys provided demographic, sexual behavior, $\mathrm{HIV} /$ syphilis infection, HIV prevention service, and testing behavior data. Results. Of 1239 participants, $15.4 \%$ were infected with HIV, incidence was 12.3 per 100 persons/year (95\% CI: 9.2-15.3), 38\% of the participants reported ever having unprotected insertive anal sex, $40 \%$ ever received free condom/lubricants in the past year, and $27.7 \%$ ever obtained free sexually transmitted infection examination/treatment in the past year. Multivariable logistic regression revealed that lower levels of HIV/AIDS related stigmatizing/discriminatory attitudes, full-time jobs, and sex debut with men at a younger age were independently associated with use of free condom/lubricants. Large social networks, higher incomes, and sexual debut with men at a younger age were associated with use of any HIV prevention and HIV testing services. Lower levels of stigmatizing/discriminatory attitudes were also associated with HIV testing. Fearing needles and being unaware of the venues for testing were top barriers for testing service utilization. Conclusion. It is imperative to address HIV/AIDS related stigmatizing/discriminatory attitudes and other barriers while delivering intervention and testing services.

\section{Introduction}

In recent years, the HIV epidemic has been rapidly expanding among men who have sex with men (MSM) in China. Male-to-male sexual contact has emerged as one of the major transmission routes for new HIV infections [1-3]. Historically, HIV-1 infection has been largely concentrated among injection drug users and former paid blood and plasma donors in geographically disparate rural areas in China $[4,5]$. However, the rapid increase of HIV-1 prevalence among MSM $[6,7]$ shows that China may be following the trajectory of other Asian countries where HIV-1 infection is a growing problem among MSM [3, 8-10].

Chongqing is one of the metropolitan cities in China with a rapid expansion of the HIV epidemic among MSM [11]. HIV prevalence among MSM in Chongqing [12] was twice 
the rates of 5-6\% among MSM from the National Sentinel Surveillance in 2011 [13]. A meta-analysis of HIV studies among MSM in low and middle-income countries estimated that globally MSM have 19.3 times higher odds of being infected with HIV compared to the general population [14]. Among an estimated 780,000 persons living with HIV/AIDS in China, nearly 55\% did not know their infection status in 2011 [3]. A recent study in Shanghai reported $28.5 \%$ of MSM engaged in sex work (money boys) and $50.5 \%$ of non-money boy MSM had never tested for HIV [15]. It is imperative to effectively reach MSM and improve the effectiveness and quality of ongoing intervention prevention services and promotion of HIV testing [16].

HIV prevention services, including the distribution of condoms, HIV testing, and testing and treatment for sexually transmitted infections (STIs), have been documented as effective measures in reducing HIV transmission [17-21]. HIV testing is the entry point to access other interventions and treatment services, while HIV infected individuals who know their infection status have been shown to reduce their risky sexual behavior and lower the risk of onward transmission [17, 19]; moreover timely treatment of HIV infected individuals may reduce community level viral load and prevent HIV transmission at the population level $[22,23]$.

Though HIV prevention services and HIV testing have been scaled up in China over the past few years, gaps in the use of these services still remain [24-26]. Limited data is available in characterizing barriers and facilitators for the use of these prevention services in different settings or regions [16].

From 2009 to 2013, three consecutive cross-sectional surveys were conducted among MSM in Chongqing using respondent driven sampling (RDS). The objective of this study was to investigate the barriers and correlates of HIV prevention services and testing behaviors among MSM in Chongqing.

\section{Methods}

2.1. Recruitment of the Participants. Three consecutive crosssectional surveys were conducted among MSM from September of 2009 to January 2010, September 2010 to January 2011, and September 2011 to January 2012. RDS was utilized to recruit the study participants. The details of the recruitment methods have been reported previously [16]. Participants were eligible if they were male, 18 years age or older, a Chongqing resident, had a history of having sex with another man in the past 12 months (sex can be defined as oral, anal, or mutual masturbation), had a valid study recruitment coupon, never participated in the survey previously, and was able to provide written informed consent. The study was approved by the Committees for Human Research of the National Center for AIDS of the China Center for Disease Control and Prevention, Vanderbilt University and the University of California San Francisco. A computer-assisted, intervieweradministrated questionnaire (CASI) was used to collect information including demographic information and sexual behaviors.
2.2. Measures. After eligibility screening and obtaining informed consent, each participant received a 20-30 minute computer-assisted interview. The questionnaire asked participants about information on demographics, sexual and drug use behaviors, HIV testing history, and stigmatizing and discriminatory attitudes towards people living with HIV/AIDS (PLWHA).

(1) Demographics and sexual risk behaviors: demographic questions included age, ethnicity, education, marital status, employment, sexual orientation, and Chongqing residence status. Risk behavior measures included ever engaging in unprotected sexual intercourse, disclosure of sexual orientation, and the number of male and female sexual partners in last 6 months.

(2) HIV prevention and HIV testing services: all participants were asked if they had participated in HIV prevention programs including receiving free condom/lubricants, free STI examination and treatment, and HIV testing. Recent testers were defined as participants who received a test for HIV in the preceding year.

(3) HIV/AIDS related stigma and discrimination: individual attitudes towards PLWHA were scored by asking participants about their agreement and disagreement ( $1=$ "yes"; 2 = "no") with 22 statements. The scale was adapted from two pilot investigations conducted in Thailand and Zimbabwe and reported elsewhere $[27,28]$. The psychometric measure includes 3 components: shame, blame and social isolation (10 items), discrimination (8 items), and equity (4 items). In our study, we included the first two components in all 3 years (equity section excluded in 2011) and therefore the first two components (18 items) were analyzed in this paper.

(4) Laboratory testing: HIV infection was screened with enzyme-linked immunosorbent assay (ELISA; Vironostika HIV Uni-Form plus O, bioMerieux, Holland) and confirmed with Western Blot test (HIV Blot 2.2 WBTM, Genelabs Diagnostics, Singapore). For HIV positives, The BED IgG-capture ELISA (BEDCEIA, Calypte Biomedical Corporation, Rockville, Maryland) was performed for all HIV-seropositive specimens. Syphilis screening was performed by rapid plasma regain (RPR; Shanghai Rongsheng, Shanghai, China) and confirmed with Treponema pallidum particle assay test (TPPA; Fujirebio Inc., Japan).

2.3. Statistical Analyses. We excluded the observations of individuals who participated in more than one round and the first observation for repeated individuals was retained in the final dataset. Since we intend to focus on service utilization analysis rather than the trend of use of these services, we believe aggregating data over three years provides a better picture of service coverage among MSM in Chongqing.

Basic demographic, risk characteristics, HIV prevention service utilization, and HIV testing factors were tabulated. 
For HIV/AIDS stigma and discrimination, scores were calculated (reverse coding items asked in the positive) for each observation within the overall scale. The factors associated with HIV prevention service utilization (three models) and recent testing (one model) were assessed with multivariable logistic regression after univariate analysis. Adjusted odds ratios (AORs) and 95\% confidence intervals (95\% CIs) were calculated in the multiple logistic regression analysis using a stepwise method. We calculated odds ratios (OR), 95\% CIs and $P$ values using bivariate logistic regression for each of these analyses. For multivariable analyses logistic regression was used to calculate adjusted odds ratios, 95\% CIs, and $P$ values. To adjust the enrollment year's impact to service use or the influence changes in time, we used recruitment year as a variable in each model. Statistical Analysis System (SAS 9.3 for Windows; SAS Institute Inc., NC, USA) software was used for all analyses.

\section{Results}

3.1. Socio-Demographics of the Study Participants. In total, we removed 163 repeated participants to obtain 1239 unique participants in the final dataset. A total of 510, 485, and 244 participants were retained in each of the three years, respectively (Table 1 ). The median age of these participants was 23 years old (interquartile (IQR): 21-27 with a range from 18 to 67$)$. Of participants, $69.6 \%$ had a college or higher level of educational attainment, nearly two-thirds currently employed with a full time job. In terms of other demographics, $30.0 \%$ were college students, more than threequarters $(78.1 \%)$ were local residents, and $7.4 \%$ reported being married to a woman. In terms of sexual orientation, $70.0 \%$ reported their sexual orientation as homosexual, while 23.9\% reported being bisexual, and $5.6 \%$ being unknown or heterosexual. A quarter $(26.3 \%)$ of men in this study reported knowing $\geq 10$ MSM and nearly $40 \%$ reported having an income of $<1000$ Yuan (CNY) per month.

3.2. Sexual Behaviors. Of participants, $8.5 \%$ reported having sex with a female partner in the past six months, $3.6 \%$ reported ever seeking sex for money or goods with male partners in the past six months, and 38\% reported having unprotected insertive anal sex.

3.3. Prevalence of HIV and Syphilis. Regarding STIs, $4.9 \%$ were infected with syphilis and $15.4 \%$ with HIV (Table 1). HIV prevalence increased from $11.6 \%$ in 2009 to $15.3 \%$ in 2010 and $23.8 \%$ in 2011. BED-CEIA estimated HIV incidence was 12.3 per 100 persons/year (95\% CI: 9.2-15.3), increasing from 7.9 in 2009 to 11.9 in 2010 and 18.2 in 2011 per 100 persons/year. Syphilis prevalence was stable at $4 \%$ to $5 \%$ across all three recruitment years.

3.4. Score of Stigmatizing and Discriminatory Attitudes Towards HIV/AIDS. The median score of stigmatizing and discriminatory attitude towards HIV/AIDS (stigma score) of all participants was $7.0($ mean $=5.9$, IQR: $3-8)$ (Table 1$)$. The average stigma score for those who received testing in the past
12 months (5.6) was lower than that for those who did not receive HIV testing $(<6.0)(P=0.03)$. The average stigma score for those who received free condom/lubricants in the past 12 months (5.4) was lower than those who did not receive HIV testing $(6.6)(P<0.01)$. The average stigma score for those who received any prevention services in the past 12 months (5.6) is lower than those who did not receive any prevention services $(<6.2)(P<0.01)$.

3.5. Received HIV Prevention and Testing Services. Only forty percent of respondents reported ever receiving condoms and/or lubricants in the past year; less than a third reported always using free condoms and being satisfied with their use, $27.7 \%$ reported ever obtained free STI examination or treatment services in the past year, and more than a third never had HIV testing in the past (Table 1). Of the participants who reported where they got free condoms and lubricants, $33.4 \%$ were from community based organizations (CBO), followed by family members $(20.9 \%)$, sex partners (15.4\%), and CDC staff (14.5\%).

\subsection{Factors Independently Associated with Receiving Preven- tion Services}

3.6.1. Factors Correlated with Receiving Free Condoms/ Lubricants. Participants who enrolled in 2009 (AOR $=0.1,95 \% \mathrm{CI}$ : 0.1-0.2, versus 2011), having a full time job $(\mathrm{AOR}=1.5,95 \%$ CI: 1.1-2.0), sexual debut with men at younger age $(\mathrm{AOR}=$ $0.7,95 \% \mathrm{CI}: 0.5-0.9,9-29$ yrs versus $\leq 18$ yrs; $\mathrm{AOR}=0.3,95 \%$ CI: $0.2-0.6 ; \geq 30$ yrs versus $\leq 18$ yrs), and lower stigma score ( $\mathrm{AOR}=0.9,95 \% \mathrm{CI}: 0.9-1.0, \mathrm{OR}$ value per scale point) were more likely to have ever obtained free condoms/lubricants in the last year Table 2, Model 1 .

3.6.2. Factors Correlated with Receiving Free STI Services. Participants who enrolled in 2009 (AOR $=0.5$, 95\% CI: 0.30.8 , versus 2011), who had larger social networks ( $\mathrm{AOR}=1.6$, 95\% CI: 1.2-2.2), those more likely to receive free STI services, those who had unprotected sex $(\mathrm{AOR}=0.7,95 \% \mathrm{CI}: 0.5-0.9)$, and those who were HIV positive (AOR = 0.6, 95\% CI: $0.4-$ 0.9 ) were less likely to receive free STI services in the past year Table 2, Model 2.

3.6.3. Factors Correlated with Receiving Any of HIV Prevention Services. Participants who enrolled in 2010 (AOR $=1.6,95 \%$ CI: 1.2-2.1, versus 2009), who had higher income (AOR $=1.5$, 95\% CI: 1.1-1.9; >1000 Yuan monthly), those with larger social networks ( $\mathrm{AOR}=1.6,95 \% \mathrm{CI}: 1.2-2.1)$, and those with sexual debut with men at a younger age $(\mathrm{AOR}=0.7,95 \% \mathrm{CI}$ : 0.5-0.9; 9-29 yrs versus $\leq 18$ yrs; AOR = 0.4, 95\% CI: 0.2-0.6; $\geq 30$ yrs versus $\leq 18$ yrs) were more likely to have ever received any of the HIV prevention services in the past year Table 2, Model 4.

3.7. Factors Independently Associated with HIV Testing. Having larger social networks $(\mathrm{AOR}=1.8,95 \% \mathrm{CI}: 1.3-2.4)$ and lower stigma scores $(\mathrm{AOR}=0.9,95 \% \mathrm{CI}: 0.9-1.0)$, enrolling in 2010, having higher income $(\mathrm{AOR}=1.7,95 \% \mathrm{CI}$ : 1.2-2.2; >1000 Yuan monthly), and being HIV-seronegative 
TABLE 1: Demographics, prevalence of HIV and Syphilis, sexual behaviors, HIV prevention, and testing mehaviors among men who have sex with men in Chongqing, China, 2009-2011.

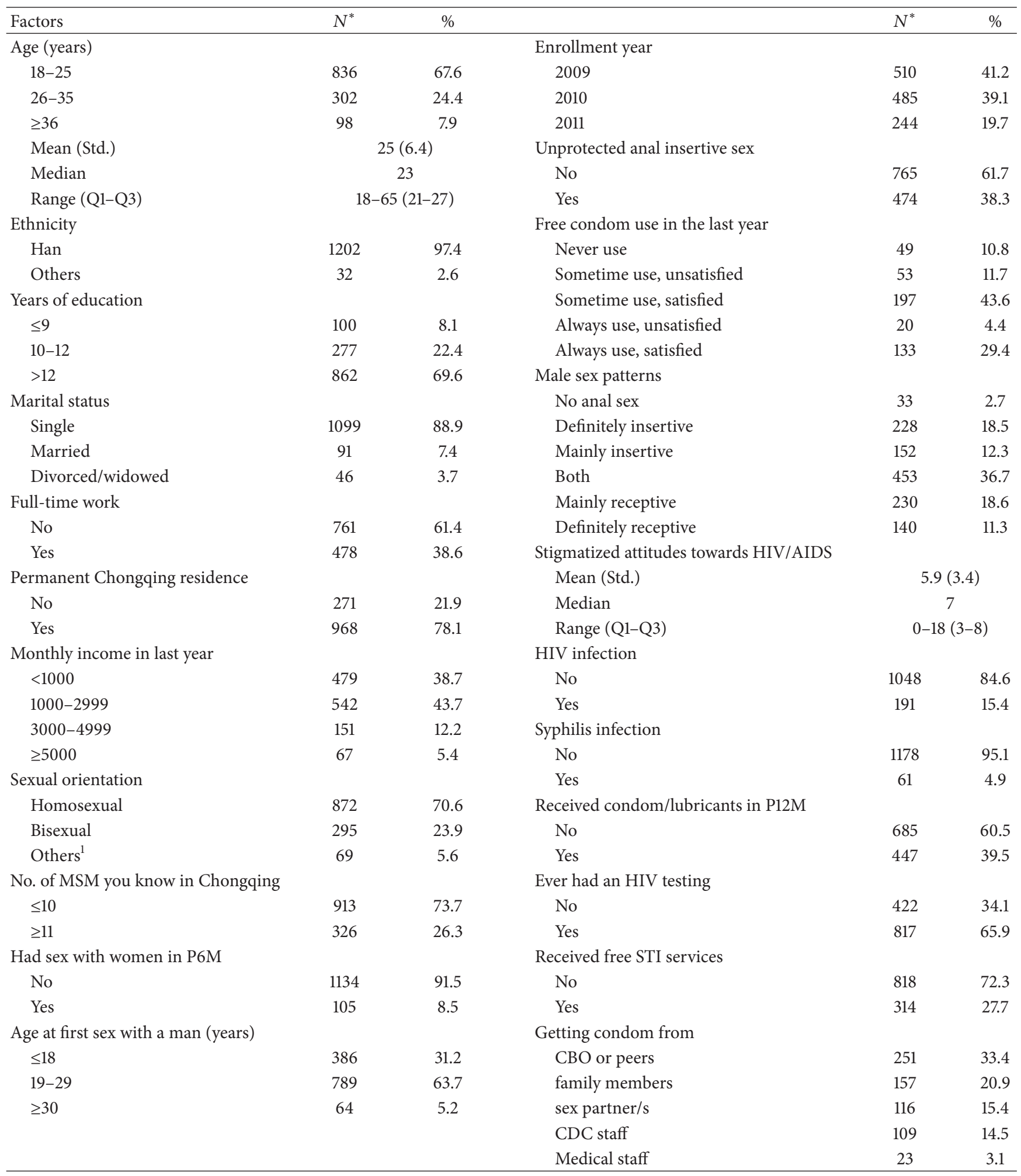

Note: P6M: the past 6 months; P12M: the past 12 months; STI: sexually transmitted infections; CBO: community-based organization; CDC: Center for Disease Control and Prevention. ${ }^{*}$ Numbers might not add to totals due to missing data. ${ }^{1}$ Others: heterosexual or unknown sexual orientation. 
TABLE 2: Factors associated with HIV prevention service and HIV testing.

\begin{tabular}{|c|c|c|}
\hline Independent factors & OR $(95 \% \mathrm{CI})$ & $\operatorname{AOR}(95 \% \mathrm{CI})$ \\
\hline \multicolumn{3}{|c|}{ Model 1: Factors associated with having ever received condom/lubricants in P12M } \\
\hline \multicolumn{3}{|l|}{ Enrollment year } \\
\hline 2010 versus 2009 & $0.9(0.7-1.2)$ & $0.9(0.7-1.2)$ \\
\hline 2011 versus 2009 & $0.1(0.0-0.1)^{\dagger}$ & $0.1(0.1-0.2)^{\dagger}$ \\
\hline Fulltime job versus part time job & $1.1(0.9-1.42)$ & $1.5(1.1-20)^{\dagger}$ \\
\hline \multicolumn{3}{|l|}{ sex debut age with a man (yrs) } \\
\hline $19-29$ versus $\leq 18$ & $0.6(0.5-0.8)^{\dagger}$ & $0.7(0.5-0.9)^{\dagger}$ \\
\hline$\geq 30$ versus $\leq 18$ & $0.3(0.2-0.6)^{\dagger}$ & $0.3(0.2-0.6)^{\dagger}$ \\
\hline No. of MSM you know in Chongqing $(\geq 11$ versus $<11)$ & $3.0(2.3-4.0)^{\dagger}$ & $2.3(1.7-3.1)^{\dagger}$ \\
\hline Stigmatizing attitudes towards HIV/AIDS* & $0.9(0.9-0.9)^{\dagger}$ & $0.9(0.9-1.0)^{*}$ \\
\hline \multicolumn{3}{|c|}{ Model 2: Factors associated with having ever receiving HIV testing in P12M } \\
\hline \multicolumn{3}{|l|}{ Enrollment year } \\
\hline 2010 versus 2009 & $2.6(2.0-3.4)^{\dagger}$ & $2.9(2.1-4.0)^{\dagger}$ \\
\hline 2011 versus 2009 & $0.3(0.2-0.5)^{\dagger}$ & $0.5(0.3-0.8)^{\dagger}$ \\
\hline \multicolumn{3}{|l|}{ Monthly income in P12M } \\
\hline $1000-2999$ versus $\leq 1000 \mathrm{CNY}$ & $1.7(1.3-2.3)^{\dagger}$ & $1.7(1.2-2.3)^{\dagger}$ \\
\hline $3000-4999$ versus $\leq 1000 \mathrm{CNY}$ & $1.3(0.9-2.0)$ & $1.3(0.9-2.1)$ \\
\hline$\geq 5000$ versus $\leq 1000 \mathrm{CNY}$ & $0.6(0.3-1.3)$ & $0.7(0.3-1.4)$ \\
\hline No. of MSM you know in Chongqing $(\geq 11$ versus $<11)$ & $2.2(1.7-2.9)^{\dagger}$ & $1.8(1.3-2.4)^{\dagger}$ \\
\hline Stigmatizing attitudes towards HIV/AIDS* & $0.9(0.9-1.0)^{*}$ & $0.9(0.9-1.0)^{*}$ \\
\hline HIV positive & $0.5(0.3-0.8)^{\dagger}$ & $0.5(0.3-0.8)^{\dagger}$ \\
\hline \multicolumn{3}{|c|}{ Model 3: Factors associated with having ever received free STI services in P12M } \\
\hline \multicolumn{3}{|l|}{ Enrollment year } \\
\hline 2010 versus 2009 & $1.3(0.9-1.7)$ & $1.2(0.9-1.6)$ \\
\hline 2011 versus 2009 & $0.6(0.4-0.8)^{\dagger}$ & $0.5(0.3-0.8)^{\dagger}$ \\
\hline No. of MSM you know in Chongqing $(\geq 11$ versus $<11)$ & $1.8(1.4-2.4)^{\dagger}$ & $1.6(1.2-2.2)^{\dagger}$ \\
\hline Unprotected insertive anal sex & $0.9(0.7-1.2)$ & $0.7(0.5-0.9)^{*}$ \\
\hline HIV positive & $0.6(0.4-0.8)^{\dagger}$ & $0.6(0.4-0.9)^{*}$ \\
\hline \multicolumn{3}{|c|}{ Model 4: Factors associated with having ever received any prevention services in P12M } \\
\hline \multicolumn{3}{|l|}{ Enrollment year } \\
\hline 2010 versus 2009 & $1.6(1.3-2.1)^{\dagger}$ & $1.6(1.3-2.1)^{\dagger}$ \\
\hline 2011 versus 2009 & $0.3(0.2-0.5)^{\dagger}$ & $0.4(0.3-0.5)^{\dagger}$ \\
\hline \multicolumn{3}{|l|}{ Monthly income } \\
\hline $1000-2999$ versus $\leq 1000 \mathrm{CNY}$ & NA & $1.5(1.1-1.9)^{\dagger}$ \\
\hline $3000-4999$ versus $\leq 1000 \mathrm{CNY}$ & NA & $1.3(0.8-1.9)$ \\
\hline$\geq 5000$ versus $\leq 1000 \mathrm{CNY}$ & NA & $1.6(0.9-2.9)$ \\
\hline No. of MSM you know in Chongqing $(\geq 11$ versus $<11)$ & $2.1(1.6-2.7)^{\dagger}$ & $1.6(1.2-2.1)^{\dagger}$ \\
\hline \multicolumn{3}{|l|}{ Sex debut age with a man (yrs) } \\
\hline $19-9$ versus $\leq 18$ & $0.7(0.6-0.9)^{\dagger}$ & $0.7(0.5-0.9)^{\dagger}$ \\
\hline$\geq 30$ versus $\leq 18$ & $0.4(0.2-0.7)^{\dagger}$ & $0.4(0.2-0.6)^{\dagger}$ \\
\hline
\end{tabular}

Note: OR: Odd ratio; 95\% CI: 95\% confidence interval; AOR: adjusted odd ratio; P6M: the past 6 months; P12M: the past 12 months; ${ }^{*} P<0.05 ;{ }^{\dagger} P<0.01$; NA: not available; all the four models were adjusted with sexual orientation, age, married status, education, employment, sex debut age with men, number of male sex partners, stigmatizing/discriminatory attitudes (consecutive variables), HIV infection status, having multiple male sex partner or not, and anal sex pattern (insertive or receptive).

(AOR $=0.5,95 \%$ CI: $0.3-0.8)$ were independently associated with receiving a test for HIV in the past year.

3.8. Barriers and Facilitators of HIV Testing. Ofparticipants, $72.1 \%$ reported "fearing needles" as the reason for not seeking a test for HIV, 66.1\% perceived they were unable to afford treatment once diagnosed as HIV positive, and $61.4 \%$ feared meeting acquaintances at testing sites (61.4\%) as reasons for not seeking an HIV test(Figure 1). While nearly two-thirds $(60.6 \%)$ of respondents perceived no risk for HIV infection, $54.5 \%$ feared knowing their HIV serostatus, 54.3\% feared to be tested due to discrimination or privacy concerns and $52.1 \%$ did not know where to get an HIV test. Regarding facilitators for HIV testing, the majority of respondents 


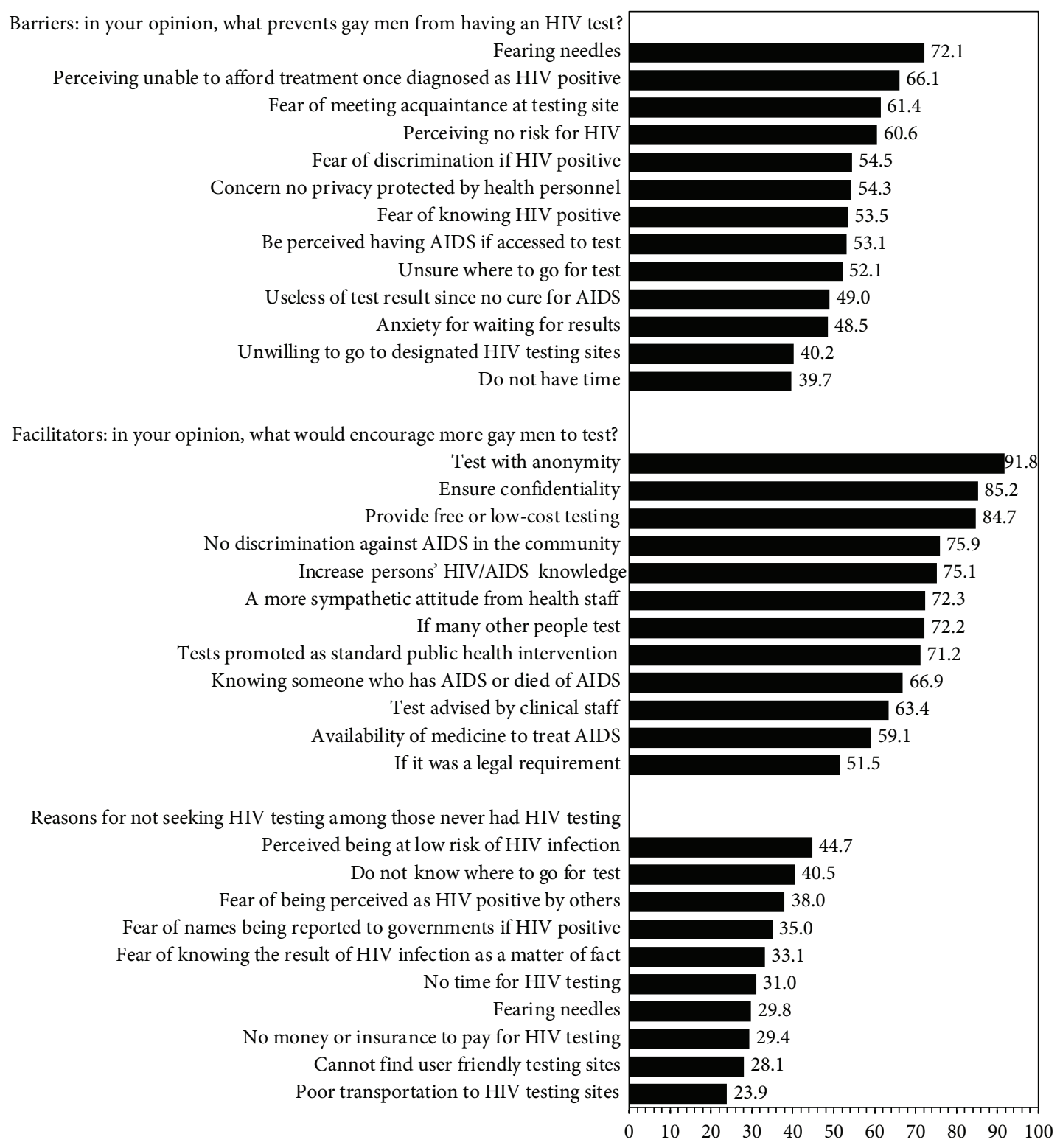

FIGURE 1: Barriers and facilitators for HIV testing and reasons for not seeking HIV testing.

(91.8\%) choose "test with anonymity," assurance of confidentiality $(85.2 \%)$, "free or low-cost testing" (84.7\%), less discrimination (75.9\%), HIV/AIDS knowledge awareness, and more sympathetic attitude from health professionals (75.1\%), as well as promoting HIV testing as part of standard HIV intervention $(71.2 \%)$ as reasons to have an HIV test. More than half $(66.9 \%)$ of respondents believed knowing somebody who had AIDS or died of AIDS might be a facilitator for HIV testing uptake.

\section{Discussion}

Statistics from the Chinese Ministry of Health and UNAIDS have shown a worrisome trend in the HIV epidemic among MSM in China $[3,10]$. Nationally, the proportion of cases attributed to MSM increased approximately 5-fold from $2.5 \%$ in 2006 to $13.7 \%$ in 2011 [3]. This suggests that the HIV epidemic is expanding rapidly in this population and that more effective prevention measures are urgently needed. The present study presents a potentially worrying scenario of rapid HIV expansion among MSM in Chongqing, with an alarming HIV prevalence, about 3 times of the national average [12] and a worrisome level of high HIV incidence. Just over half of respondents in Chongqing received at least one HIV prevention service recently, including free condoms/lubricants, free HIV testing, and free STIs examination in the last year. Only a quarter of MSM had a test for HIV recently, while a third had never tested for HIV. This study highlights that HIV/AIDS related stigmatizing and discriminatory attitudes and a variety of barriers must be 
addressed in order to improve the delivery of prevention services and to expand HIV testing effectively.

Studies $[27,28]$ have shown that social stigma and punitive civil environments may lead to delays in seeking HIV and STI testing, and subsequent initiation of antiretroviral therapy [18]. Our previous report suggested that stigma and discriminatory attitudes were associated with not seeking HIV testing [28]. The present study suggests that stigma and discrimination are not only associated with HIV testing but are also associated with HIV prevention services, especially the use of free condom/lubricants. This is consistent with the finding from the barriers and facilitators assessment in present study. More than half of participants listed "fear of meeting acquaintance at testing site," "fear of discrimination if being HIV positive," and "perceived to be living with HIV/AIDS if initially take HIV test," as discrimination and stigma related barriers. A recent qualitative study in China suggested that reducing HIV-related stigma and discrimination can actually increase HIV testing and relevant HIVservice uptake [29].

The present study also found the two main facilitators for HIV testing are "testing with anonymity" followed by "assurance of confidentiality." This finding is consistent with another study that those recruited via social networks (who benefit developing the supportive relationships from group membership) prefer to maintain their anonymity [30]. Unwillingness to go to an HIV clinic was an additional barrier to testing. Financial assistance, transportation support, and mobile testing vans should be considered to address this situation, for example, conducting HIV testing in community venues frequented by MSM. For persons using clinic-based testing, high quality counseling, and nonjudgmental friendly care and service providers can help clients reduce their fear and concerns and facilitate the linkage of seropositive persons to care. Along with expanded education and social marketing, a welcoming and nonjudgmental environment for HIV testing is needed [31].

The barriers and facilitators assessment revealed that fear of needles was the first perceived barrier against taking an HIV test, followed by fear of cost of testing. This finding supports the use of the rapid oral test (ROT) or other noninvasive HIV testing method for testing promotion. China National Guideline for Detection of HIV/AIDS in 2009 [32] recommended saliva based oral rapid HIV testing for use in clinics or Emergency Department. It is necessary to provide training for the use of ROT in community based organizations $(\mathrm{CBO})$ or in grass-root level health agencies.

More than half of the participants reported one of the barriers to HIV testing as not knowing where to get a test. Another important barrier was the perception of being not able to afford HIV testing and/or treatment. This finding further underscored the gap in promoting HIV testing among MSM $[33,34]$ and the needs to address various barriers in delivering prevention and HIV testing services.

The age of participants in the present study is younger compared with other reports $[29,35]$, while one predictor for free condom/lubricants accessibility in last year was sexual debut with men at a younger age. Similarly with Choi's study
[5], the present study found that the younger age of sexual debut with men was associated with larger size of social networks $(r=-0.15)$ and larger social networks were associated with the use of HIV prevention services. However, our findings differ with another report that suggests MSM sexual debut predicted more frequently change of sex partners and other risk behaviors [36]. The finding of our study leads us to believe that the larger size of an individual's social network might be an advantage for the use of HIV prevention services. While RDS was used to recruit participants for research [37, 38 ] it could also be applied as a mechanism to deliver intervention services among Chinese MSM as it has been used among intravenous drug users (IDU) [39, 40]. Younger MSM may be more intensely engaging in dynamic social networks where individuals interact often with large numbers of peers. Along with more intensity in dynamic cyber life via emerging media, Internet based social network, for example, "Weibo" (microblog, a "Facebook" like social network in China), QQ, email, and other "text message" or instant messaging "Apps" via mobile phone/Internet could serve as effective channels to promote education message for intervention services delivering.

MSM in our study who had full time jobs were more likely to receive condoms and/or lubricants, which corroborated with a recent report in Beijing that lack of stable employment and stable income leads to vulnerabilities to STI infection [35]. Our study shows MSM were more likely to utilize the services in 2010 compared to 2009 but less likely to use services in 2011 compared to 2009. The reason might be due to the RDS recruitment. Along with the longer referral chains, persons enrolled later, who were more hidden, may have been less likely to be reached by the prevention programs. Another possible reason for the peak of service utilization appearing in 2010 rather than 2011 could be that the ChinaGlobal Fund AIDS Program delayed funds disbursement and made CBO face the funds vacuum for almost 1 year period since 4thquarter of 2010 to quarter 3rd of 2011 [41]. The delay exposed vulnerability in $\mathrm{CBO}$ 's sustainability and lack of monetary incentives resulted in their inactivity in HIV prevention service delivery.

Unprotected receptive anal sex among MSM is the sexual behavior with the highest risk for HIV transmission [4246]. Nondisclosure of HIV status with casual partners has been associated with sexual transmission. Not knowing a partner's HIV status and not communicating HIV status were particularly common in the casual partnerships. Efforts to improve communication skills to disclose HIV status and use condoms with sexual partners might reduce the sexual transmission of HIV among MSM [47, 48]. However, almost half $(48.2 \%)$ of the men who tested positive for HIV during the survey were unaware of their infection, highlighting the importance of consistent and correct condom use in the prevention of HIV transmission. Therefore, monitoring the prevalence of risky sexual behaviors and identifying HIV infected individuals who do not know their status could provide valuable information for prevention programs, which are critical to reduce sexual transmission of HIV among MSM. 
Multifaceted comprehensive interventions should be implemented. Infrastructure and healthy social norm building (e.g., normalizing condom use and routine HIV testing) should be part of the comprehensive approach. Infrastructure building could be considered such as using mobile vans or equipping health professionals and local CBOs serving local MSM community to promote HIV testing. Healthy social norm building can be achieved by diffusing most relevant health information via available and accessible social media like websites or text messages to promote testing in Chinese MSM $[31,49,50]$. The extent of the risk of acquiring HIV in the United States today is largely defined by a person's sexual network rather than his or her individual behaviors [51]. Group intervention with targeting the behaviors and beliefs from community perspective might be promising as an avenue for prevention in China [52]. For example, collaborating with event promoters offers valuable opportunities to provide condoms, lubricants, and HIV/STI testing [53].

We recognized the limitations of this study. First, selfreported information about the sexual behavior and service uptake may lead to recall bias. Second, because the information was collected by interviewers, some behaviors might have been underreported or over reported. For example, participants might have underreported socially undesirable behaviors (e.g., drug uses) or might have overreported socially desirable behaviors, (e.g., condom use or HIV testing). Third, the nature of the cross-sectional study design precluded the ascertainment of casual relationship. Forth, the study sample comprised of almost one-third college students, which raises the concern of the representativeness of the sample [54]. In addition, the inclusion criteria of willingness to provide a blood specimen may have led to potential under participation in the survey thus increasing selection bias. Despite these limitations, we believe that the present study generated valuable information to enhance the effectiveness of HIV prevention programs among the MSM community.

In summary, the present study revealed that an alarming HIV epidemic is occurring among MSM in Chongqing. Various barriers to the use of HIV prevention service and HIV testing among this population still remain. It is imperative to address the stigmatizing/discriminatory attitudes and other barriers while delivering prevention services and promoting HIV testing.

\section{Ethical Approval}

The study protocol was approved by institutional review boards of the National Center for HIV/AIDS Control and Prevention, China CDC, the Vanderbilt University, and the University of California San Francisco in the USA.

\section{Consent}

Informed patient consent was obtained.

\section{Conflict of Interests}

The authors declare that there is no conflict of interests regarding the publication of this paper.

\section{Authors' Contribution}

Dayong Huang, Yifei $\mathrm{Hu}$, and Guohui Wu contributed equally to this work. Dayong Huang, Yifei Hu, and Yujiang Jia conceptualized the paper, did analysis, and drafted the initial version of the paper. Yuhua Ruan and Yan Xiao are the principal investigator of the research. Jiangping Sun, Yuhua Ruan, Yan Xiao and Yujiang Jia, H. F. Raymond, Willi McFarland, and Wei Ma provided guidance on study design implementation, data analysis, and paper writing. Guohui $\mathrm{Wu}$ and Rongrong Lu conducted questionnaire interviews. Yujiang Jia, H. F. Raymond, and Jiangping Sun contributed to the revision of the paper. All authors read and approved the final version of the paper.

\section{Acknowledgments}

This study was supported by Grants from the National Natural Science Foundation of China (81273188), the Ministry of Science and Technology of China (2012ZX10001002), Chinese State Key Laboratory for Infectious Disease Development Grant (2012SKLID103), and the Fogarty International Clinical Research Scholars-Fellows Support Center at Vanderbilt (Grant no. R24TW007988).

\section{References}

[1] E. P. F. Chow, D. P. Wilson, and L. Zhang, "Patterns of condom use among men who have sex with men in China: a systematic review and meta-analysis," AIDS and Behavior, vol. 16, no. 3, pp. 653-663, 2012.

[2] M. Han, L.-G. Feng, Y. Jiang et al., "Surveillance on HIV-1 incidence among men who have sex with men in Chongqing, China, 2006-2008," Zhonghua Liu Xing Bing Xue Za Zhi, vol. 30, no. 9, pp. 878-881, 2009.

[3] China MoHotPsRo, "2012 China AIDS Response Progress Report," 2012.

[4] S. L. Chen, H. R. Zhao, Y. Q. Zhang et al., "A retrospective cohort study on the natural history of AIDS caused by blood transfusion," Zhonghua Liu Xing Bing Xue Za Zhi, vol. 33, no. 7, pp. 658662, 2012.

[5] B. Shao, W. J. Li, T. Liu et al., "Subtype B was the dominant strain among HIV type 1 infections except for the population of men who have sex with men in Harbin City, China," AIDS Research and Human Retroviruses, vol. 29, no. 9, pp. 1260-1264, 2013.

[6] W. Lin, S. Chen, N. Seguy et al., "Is the HIV sentinel surveillance system adequate in China? Findings from an evaluation of the national HIV sentinel surveillance system," Western Pacific Surveillance and Response Journal, vol. 3, no. 4, pp. 76-85, 2012.

[7] Y. Xu, Z. Zhang, D. Li et al., "Willingness to use the oral fluid HIV rapid test among men who have sex with men in Beijing, China," PLoS ONE, vol. 8, no. 5, Article ID e64652, 2013.

[8] C. Beyrer and Q. Abdool Karim, "The changing epidemiology of HIV in 2013," Current Opinion in HIV and AIDS, vol. 8, no. 4, pp. 306-310. 
[9] F. van Griensven, J. W. de Lind van Wijngaarden, S. Baral, and A. Grulich, "The global epidemic of HIV infection among men who have sex with men," Current Opinion in HIV and AIDS, vol. 4, no. 4, pp. 300-307, 2009.

[10] J. Bridge, J. V. Lazarus, and R. Atun, "HIV epidemics and prevention responses in Asia and Eastern Europe: lessons to be learned?” AIDS, vol. 24, no. 3, pp. S86-S94, 2010.

[11] L.-G. Feng, M.-J. Wang, M. Han, X.-B. Ding, and Y. Jiang, "Drug resistance among recent HIV-1 infected men who have sex with men in Chongqing municipality of China," Zhonghua Liu Xing Bing Xue Za Zhi, vol. 29, no. 5, pp. 455-458, 2008.

[12] Y. Zhang, P. Chen, R. Lu et al., "Prevalence of HIV among men who have sex with men in Chongqing, China, 2006-2009: cross -sectional biological and behavioural surveys," Sexually Transmitted Infections, vol. 88, no. 6, pp. 444-450, 2012.

[13] China MoHotPsRo, "China AIDS Response Progress Report. 2012," 2012.

[14] S. Baral, F. Sifakis, F. Cleghorn, and C. Beyrer, "Elevated risk for HIV infection among men who have sex with men in lowand middle-income countries 2000-2006: a systematic review," PLoS Medicine, vol. 4, no. 12, pp. 1901-1911, 2007.

[15] Z. J. Huang, N. He, E. J. Nehl et al., "Social network and other correlates of HIV testing: findings from male sex workers and other MSM in Shanghai, China," AIDS and Behavior, vol. 16, no. 4, pp. 858-871, 2012.

[16] W. Ma, H. F. Raymond, E. C. Wilson et al., "Participation of HIV prevention programs among men who have sex with men in two cities of China-a mixed method study," BMC Public Health, vol. 12, p. 847, 2012.

[17] V. D. Lima, K. Johnston, R. S. Hogg et al., "Expanded access to highly active antiretroviral therapy: a potentially powerful strategy to curb the growth of the HIV epidemic," Journal of Infectious Diseases, vol. 198, no. 1, pp. 59-67, 2008.

[18] K. H. Mayer, D. P. Wheeler, L. G. Bekker et al., "Overcoming biological, behavioral, and structural vulnerabilities: new directions in research to decrease HIV transmission in men who have sex with men," Journal of Acquired Immune Deficiency Syndromes, vol. 63, supplement 2, pp. S161-S167, 2013.

[19] S. H. Vermund, S. L. Hodder, J. E. Justman et al., "Addressing research priorities for prevention of HIV infection in the United States," Clinical Infectious Diseases, vol. 50, no. 3, pp. S149-S155, 2010.

[20] H. Grosskurth, F. Mosha, J. Todd et al., "Impact of improved treatment of sexually transmitted diseases on HIV infection in rural Tanzania: randomised controlled trial," The Lancet, vol. 346, no. 8974, pp. 530-536, 1995.

[21] B. M. Branson, H. H. Handsfield, M. A. Lampe et al., "Revised recommendations for HIV testing of adults, adolescents, and pregnant women in health-care settings," MMWR. Recommendations and Reports, vol. 55, no. RR-14, pp. 1-17, 2006, quiz CE1114.

[22] M. S. Cohen, Y. Q. Chen, M. McCauley et al., "Prevention of HIV-1 infection with early antiretroviral therapy," The New England Journal of Medicine, vol. 365, no. 6, pp. 493-505, 2011.

[23] J. S. Montaner, V. D. Lima, R. Barrios et al., "Association of highly active antiretroviral therapy coverage, population viral load, and yearly new HIV diagnoses in British Columbia, Canada: a population-based study," The Lancet, vol. 376, no. 9740, pp. 532539, 2010.

[24] M. Y. Chen, J. E. Bilardi, D. Lee, R. Cummings, M. Bush, and C. K. Fairley, "Australian men who have sex with men prefer rapid oral HIV testing over conventional blood testing for HIV,"
International Journal of STD and AIDS, vol. 21, no. 6, pp. 428430, 2010.

[25] T. Sanchez, T. Finlayson, A. Drake et al., "Human immunodeficiency virus (HIV) risk, prevention, and testing behaviorsUnited States, National HIV Behavioral Surveillance System: men who have sex with men, November 2003-April 2005," MMWR. Surveillance Summaries, vol. 55, no. 6, pp. 1-16, 2006.

[26] A. M. Oster, I. W. Miles, B. C. Le et al., "HIV testing among men who have sex with men-21 cities, United States, 2008," Morbidity and Mortality Weekly Report, vol. 60, no. 21, pp. 694-699, 2011.

[27] B. L. Genberg, S. Kawichai, A. Chingono et al., "Assessing HIV/ AIDS stigma and discrimination in developing countries," AIDS and Behavior, vol. 12, no. 5, pp. 772-780, 2008.

[28] X. Li, H. Lu, X. Ma et al., "HIV/AIDS-related stigmatizing and discriminatory attitudes and recent HIV testing among men who have sex with men in Beijing," AIDS and Behavior, vol. 16, no. 3, pp. 499-507, 2012.

[29] C. Wei, H. Yan, C. Yang et al., "Accessing HIV testing and treatment among men who have sex with men in China: a qualitative study," AIDS Care, vol. 26, no. 3, pp. 372-378, 2014.

[30] J. L. Katz, E. R. Orellana, D. D. Walker, L. Viquez, J. F. Picciano, and R. A. Roffman, "The sex check the development of an HIVprevention service to address the needs of Latino MSM," Journal of Gay and Lesbian Social Services, vol. 18, no. 1, pp. 37-49, 2006.

[31] L. Zhang, Y. Xiao, R. Lu et al., "Predictors of HIV testing among men who have sex with men in a large Chinese city," Sexually Transmitted Diseases, vol. 40, no. 3, pp. 235-240, 2013.

[32] National Center for HIV/AIDS Control and Prevention CC, "National Guideline for Detection of HIV/AIDS," 2009.

[33] W. Guo, A.-J. Song, X.-H. Zhao, H.-D. Meng, and Z.-Y. Wu, "A qualitative investigation of the usage of VCT service among men who have sex with men," Chinese Journal of Health Education, vol. 24, no. 4, pp. 276-278, 2008.

[34] G. Li, Z. Zhang, W. Shi et al., "Current situation analysis on the utilization and demand of VCTservice among MSM group," Modern Preventive Medicine, vol. 36, no. 18, pp. 3555-3559, 2009.

[35] Y. Hu, H. Z. Qian, J. Sun et al., “Anal human papillomavirus infection among HIV-infected and uninfected men who have sex with men in Beijing, China," Journal of Acquired Immune Deficiency Syndromes, vol. 64, no. 1, pp. 103-114, 2013.

[36] A. Y. Outlaw, G. Phillips, L. B. Hightow-Weidman et al., "Age of MSM sexual debut and risk factors: results from a multisite study of racial/ethnic minority YMSM living with HIV," AIDS Patient Care and STDs, vol. 25, no. 1, pp. S23-S29, 2011.

[37] S. D. Young, W. G. Cumberland, S. J. Lee, D. Jaganath, G. Szekeres, and T. Coates, "Social networking technologies as an emerging tool for HIV prevention: a cluster randomized trial," Annals of Internal Medicine, vol. 159, no. 5, pp. 318-324, 2013.

[38] W. Guo, S. Bao, W. Lin et al., "Estimating the size of HIV key affected populations in Chongqing, China, using the network scale-up method," PLoS ONE, vol. 8, no. 8, Article ID e71796, 2013.

[39] R. Magnani, K. Sabin, T. Saidel, and D. Heckathorn, "Review of sampling hard-to-reach and hidden populations for HIV surveillance," AIDS, vol. 19, no. 2, pp. S67-S72, 2005.

[40] J. L. Wylie and A. M. Jolly, "Understanding recruitment: outcomes associated with alternate methods for seed selection in respondent driven sampling," BMC Medical Research Methodology, vol. 13, no. 1, p. 93, 2013. 
[41] Chongqing-CDC, "Anual Report of Chongqing HIV Prevention and Treatment," 2013.

[42] B. A. Koblin, M. J. Husnik, G. Colfax et al., "Risk factors for HIV infection among men who have sex with men," AIDS, vol. 20, no. 5, pp. 731-739, 2006.

[43] M. L. Ekstrand, R. D. Stall, J. P. Paul, D. H. Osmond, and T. J. Coates, "Gay men report high rates of unprotected anal sex with partners of unknown or discordant HIV status," AIDS, vol. 13, no. 12, pp. 1525-1533, 1999.

[44] S. E. Morin, W. T. Steward, E. D. Charlebois et al., "Predicting HIV transmission risk among HIV-infected men who have sex with men: findings from the healthy living project," Journal of Acquired Immune Deficiency Syndromes, vol. 40, no. 2, pp. 226235, 2005.

[45] S. Y. Chen, S. Gibson, M. H. Katz et al., "Continuing increases in sexual risk behavior and sexually transmitted diseases among men who have sex with men: San Francisco, Calif, 1999-2001," American Journal of Public Health, vol. 92, no. 9, pp. 1387-1388, 2002.

[46] N. Crepaz, G. Marks, A. Liau et al., "Prevalence of unprotected anal intercourse among HIV-diagnosed MSM in the United States: a meta-analysis," AIDS, vol. 23, no. 13, pp. 1617-1629, 2009.

[47] F. Molitor, M. Facer, and J. D. Ruiz, "Safer sex communication and unsafe sexual behavior among young men who have sex with men in California," Archives of Sexual Behavior, vol. 28, no. 4, pp. 335-343, 1999.

[48] M. A. Chesney, B. A. Koblin, P. J. Barresi et al., "An individually tailored intervention for HIV prevention: baseline data from the EXPLORE study," American Journal of Public Health, vol. 93, no. 6, pp. 933-938, 2003.

[49] S. M. Noar, "Computer technology-based interventions in HIV prevention: state of the evidence and future directions for research," AIDS Care, vol. 23, no. 5, pp. 525-533, 2011.

[50] C. M. Khosropour, B. A. Johnson, A. V. Ricca, and P. S. Sullivan, "Enhancing retention of an Internet-based cohort study of men who have sex with men (MSM) via text messaging: randomized controlled trial," Journal of Medical Internet Research, vol. 15, no. 8, p. e194, 2013.

[51] W. M. El-Sadr, K. H. Mayer, and S. L. Hodder, "AIDS in America -forgotten but not gone," The New England Journal of Medicine, vol. 362, no. 11, pp. 967-970, 2010.

[52] D. Kang, X. Tao, M. Liao et al., "An integrated individual, community, and structural intervention to reduce HIV/STI risks among female sex workers in China," BMC Public Health, vol. 13, p. 717, 2013.

[53] C. Grov, H. J. Rendina, A. S. Breslow, A. Ventuneac, S. Adelson, and J. T. Parsons, "Characteristics of men who have sex with men (MSM) who attend sex parties: results from a national online sample in the USA," Sexually Transmitted Infections, vol. 90, no. 1, pp. 26-32, 2013.

[54] L. Vu, K. Andrinopoulos, W. Tun, and S. Adebajo, "High levels of unprotected anal intercourse and never testing for HIV among men who have sex with men in Nigeria: evidence from a cross-sectional survey for the need for innovative approaches to HIV prevention," Sexually Transmitted Infections, vol. 89, no. 8, pp. 659-665, 2013. 


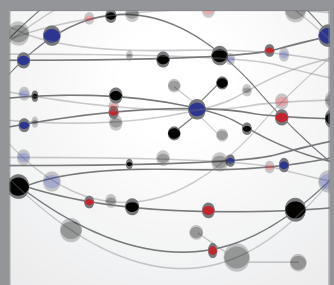

The Scientific World Journal
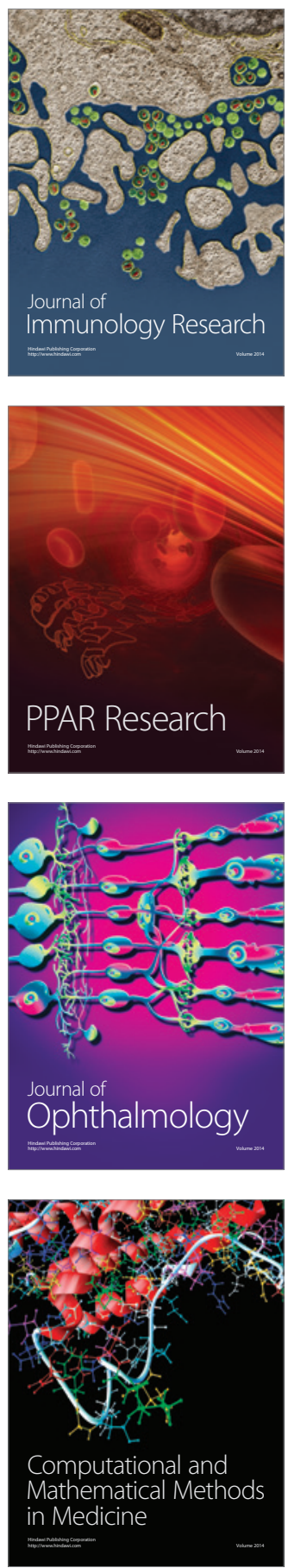

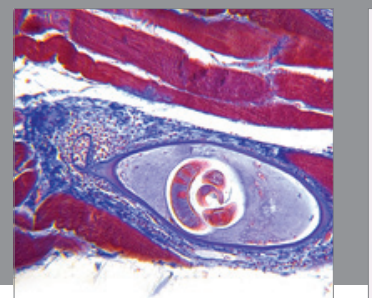

Gastroenterology

Research and Practice
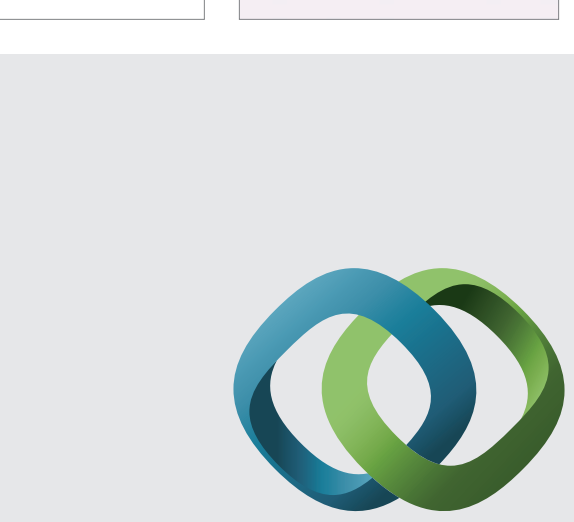

\section{Hindawi}

Submit your manuscripts at

http://www.hindawi.com
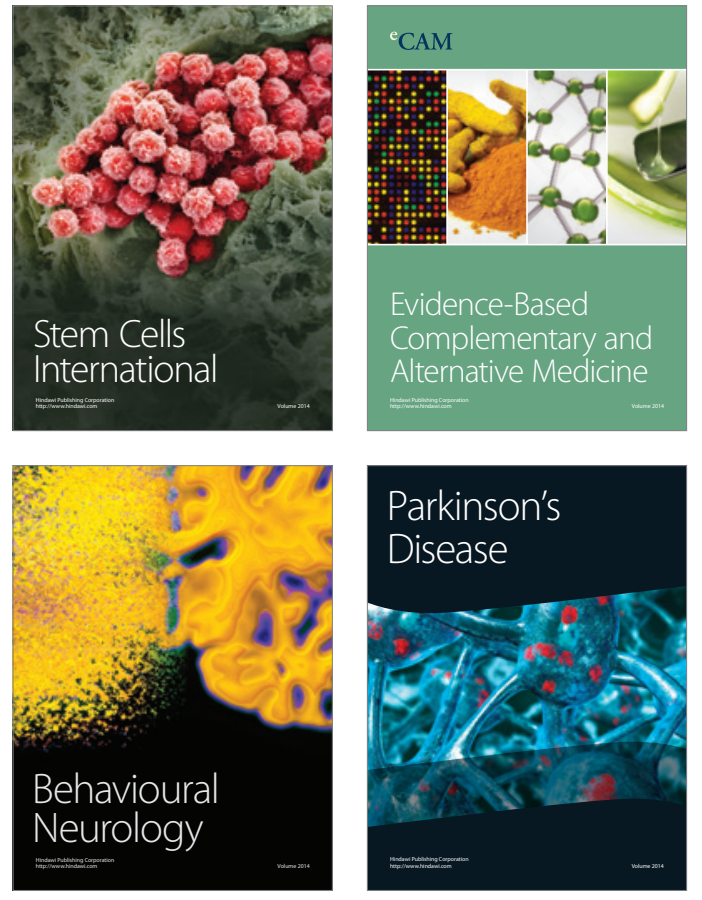
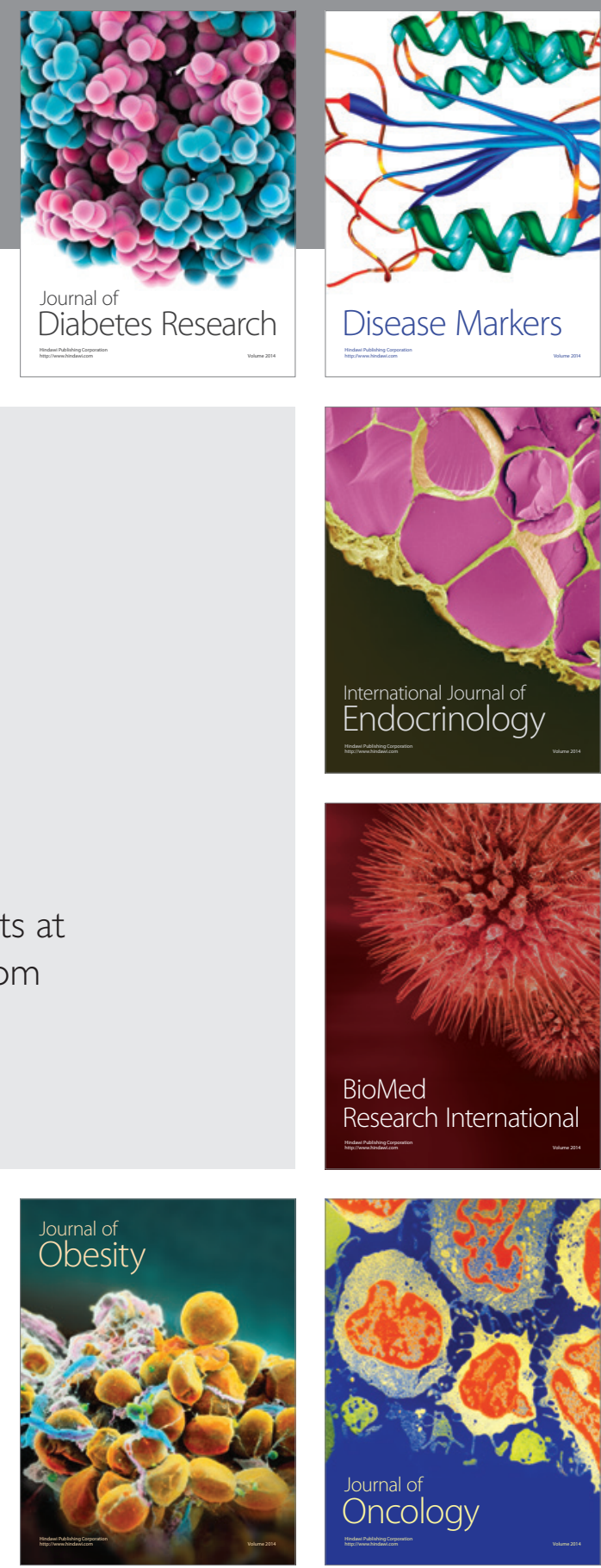

Disease Markers
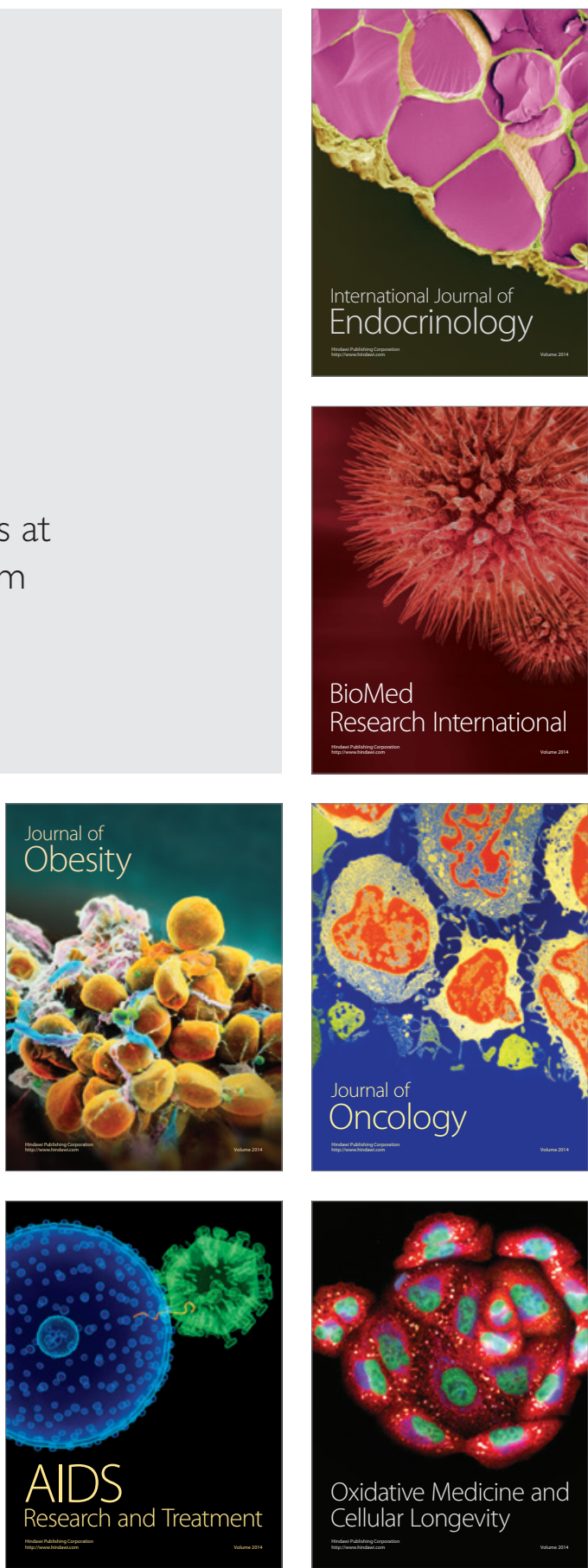